\title{
Cochlear implantation in patients with acute or chronic middle ear infectious disease: a review of the literature
}

\author{
Catharine A. Hellingman · Erwin A. Dunnebier
}

Received: 12 December 2007 / Accepted: 25 September 2008 / Published online: 14 October 2008

(C) The Author(s) 2008. This article is published with open access at Springerlink.com

\begin{abstract}
Although in the past cochlear implantation was considered contraindicated in patients with acute (AOM) or chronic suppurative otitis media (CSOM) with or without middle ear cholesteatoma, recent developments now make it possible to perform cochlear implantation in these patients. Various procedures are available to make the ears of patients with either acute or CSOM suitable for cochlear implantation and to minimize the risk of recurrence of the disease, device extrusion, or intracranial complications. This review discusses these different approaches for optimizing implant survival and preventing complications related to otitis media. We performed a comprehensive literature search of the MEDLINE database. Cochlear implantation can be safely performed in patients with otitis media. However, the infection should be adequately controlled well before implantation, and all measures should be taken to prevent recurrent disease. Therefore, the procedure used should be tailored to individual clinical findings. This article provides a guideline to optimize the course of action in patients suffering from AOM, CSOM or their sequelae in preparation for cochlear implantation.
\end{abstract}

Keywords Cochlear implantation - Otitis media . Tympanic membrane perforation - Radical cavity . Cholesteatoma

C. A. Hellingman · E. A. Dunnebier $(\bowtie)$

Department of Otorhinolaryngology,

University Medical Center Utrecht, Postbox 85500,

3508 GA Utrecht, The Netherlands

e-mail: e.a.dunnebier@umcutrecht.nl

\section{Introduction}

Acute otitis media (AOM) is the most common pediatric infection, with an incidence of $60-80 \%$ in the first 6 years of life and a high risk of recurrence. Therefore, many young deaf patients are eligible to receive a cochlear implant suffer from otitis media. Chronic suppurative otitis media (CSOM) can cause several problems, beside chronic otorrhea, that may give rise to sensorineural hearing loss, and thus may be a coincidental finding or the cause of deafness in patients requiring a cochlear implant (CI).

In the past, cochlear implantation was contraindicated in patients with otitis media [1], because of the risk of meningitis, tympanic membrane perforation, recurrent cholesteatoma, and extrusion of the electrode in a radical cavity because of breakdown of the thin epithelial lining. Moreover, the development of otitis media after implantation may cause intracranial complications or device extrusion or necessitates device removal. Despite these potential problems, there is sometimes no choice but to perform cochlear implantation in an "infected" ear; for example, if the patient has bilateral otitis media or if the patient has one-sided otitis media but the other ear is unsuitable for device implantation because of residual hearing, anatomical variations, or obstruction of the cochlear lumen. A pre-operative CT-scan is made to evaluate cochlear patency and anatomical malformations. Furthermore, this CT-scan may provide additional information about the pathology, as discussed in this article. MRI will be complementary to detect or eliminate partial or total obliteration of the cochlear duct due to fibrosis or ossifications.

The aim of this article was to review the literature on cochlear implantation in relation to acute and CSOM and its consequences. We discuss the different approaches used for optimizing implant survival and for preventing complications associated with otitis media. 


\section{Review of the literature}

We performed a comprehensive literature search of the MEDLINE database, using the following key words: cochlear implantation or cochlear implant and otitis media or CSOM or tympanic membrane perforation or radical cavity or cholesteatoma. We selected relevant articles based on the articles' title and abstract.

\section{Acute otitis media}

Acute otitis media (AOM) is the most common bacterial infection among children; by 3 years of age, $50-85 \%$ of children have had AOM [2]. Moreover, recurrent infections are common, affecting $10-20 \%$ of children by age 1 year [2]. Therefore, an acute infection or a recent history of (recurrent) $\mathrm{OM}$ is observed relatively frequent in children who require a CI, especially in early implantation. Although in the past, $\mathrm{OM}$ was considered a risk factor for complications after implantation, delaying cochlear implantation in OM-prone children until they have outgrown their susceptibility to otitis media has significant repercussions on speech-language and educational development [2]. Recent reports have demonstrated that cochlear implantation can be performed safely in patients with a history of AOM and that a significant delay is not warranted in these patients [3-5]. A prospective study [4,5], with a cohort of 60 children, of which 34 were classified as OM-prone, demonstrated that if control of $\mathrm{OM}$ is achieved before cochlear implantation, the incidence of AOM decreases post-implantation. Moreover, no complications were seen in the OM-prone children receiving a cochlear implant, even in the children suffering an episode of AOM post-implantation.

It should be emphasized that in all these studies [3-5], the infection was under control before cochlear implantation. If this is not the case, an increased risk of bacterial contamination of the device or meningitis could be expected. This control of AOM before implantation can be achieved conservatively with pain medication or antibiotics. However, in patients with a recent history of recurrent $\mathrm{OM}$ or in children with a high risk of recurrence due to their age, ventilating tubes may be more effective than antibiotics. Additional treatments, such as adenoidectomy, may also be of use [3-6]. Patients with a dry, clean ventilation tube in place can receive an implant without further delay [6].

If it is adequately controlled, (recurrent) AOM should not be considered a contraindication for cochlear implantation, and implantation in such children should not be postponed.

\section{Tympanic membrane perforation}

The tympanic membrane may be perforated, without signs of inflammation, in patients with a history of either acute or chronic otitis media. In the case of a simple, dry, tympanic membrane perforation, several authors recommend repair of the perforation first, followed by cochlear implantation, if the drum has been intact for at least 3 months [7-10]. However, these studies retrospectively described only six patients in total with a tympanic membrane perforation. Myringoplasty performed simultaneously with cochlear implantation would seem the most straightforward procedure, but repair of the tympanic membrane perforation occasionally requires more than one procedure [7], and myringoplasty failure would leave the electrode vulnerable to the external environment. Therefore, some authors have used other techniques in a single-stage procedure with cochlear implantation, such as meatal closure [11-13] or radical mastoidectomy with complete obliteration of the cavity $[8,14]$. Meatal closure carries the disadvantage that otoscopic visualization is not possible in these patients, and thus early diagnosis and treatment of infection, which is necessary to minimize the risk of meningitis, is more difficult.

We suggest that a distinction should be made between small and large perforations. As patients with a dry, clean ventilation tube, in place can undergo cochlear implantation (without prior tube removal) without an increased risk of complications [6], it would appear safe to place an implant when there is a persistent small tympanic membrane perforation. Therefore, such patients do not need to undergo myringoplasty followed by device implantation during a later procedure. However, if the perforation is large, the electrode is more vulnerable to the external environment, which increases the likelihood of infection or damage. Therefore, it is always appropriate to protect the electrode if there is a large perforation. If myringoplasty is unsuccessful, one option is to repeat the procedure and to delay cochlear implantation by at least another 3 months, to allow the perforation to close or at least become smaller. Another option is to perform either meatal closure or radical mastoidectomy in conjunction with cochlear implantation, but this complex procedure is associated with additional complications. It should be borne in mind that closure of the perforation, to protect the electrode array in the middle ear, might reactivate the CSOM. This makes it inadvisable, even if the perforation is small, to perform myringoplasty and cochlear implantation at the same time.

Therefore, in patients with a dry tympanic membrane perforation, repair of the perforation and cochlear implantation in a staged procedure appears to be the best solution. In the case of chronic purulent discharge from a tympanic membrane perforation, the ear should be treated as any ear with active CSOM, as mentioned later.

\section{CSOM or cholesteatoma}

Cochlear implantation in patients with persistent purulent discharge from an ear because of CSOM, middle ear 
cholesteatoma, or both, is a challenge. The incidence of (the sequelae of) CSOM, active and inactive, in patients receiving a $\mathrm{CI}$ is relatively rare, and is highly dependent on institutional policies $(2.2-10.9 \%)$ [7, 8, 17]. Therefore, the exact risk of complications in these patients is not known. Although only few documented cases exist $[15,16]$, there is a potential danger of device extrusion, meningitis, and other intracranial complications in these patients. Therefore, it is essential to completely eradicate the disease before implantation, to prevent recurrent or residual infection or cholesteatoma in the implanted ear. Donnelly et al. [15] describe three patients with a history of CSOM who underwent mastoidectomy before implantation, all suffering from recurrent CSOM post-implantation [15]. Therefore, conservative management, such as antibiotics, and conventional surgical management, such as ventilating tubes, mastoidectomy, or tympanoplasty, does not seem to sufficiently eliminate the risk of recurrence. Especially in case of cholesteatoma, intact canal wall tympano mastoidectomy or radical mastoidectomy is the most thorough technique to clear the ear of disease and are recommended for this reason $[7,8,10,17,18]$. Four retrospectively conducted studies describe in total 14 patients with active CSOM, with or without cholesteatoma and with or without previous mastoidectomy, in which this procedure was carried out without complications post-implantation [7, 8, 17, 18].

Most surgeons prefer a staged procedure for implantation in patients with purulent CSOM or cholesteatoma [7, 8, 10, 14, 17-21]. In the case of CSOM, a CI can be placed after a delay of 3-6 months if the disease is eradicated [7, 18, 19]. The presence of cholesteatoma should be re-evaluated after at least 6-12 months and, therefore, cochlear implantation must be delayed by the same time [7, 18-20]. This means that implantation is significantly delayed, especially if the ear is not healthy after the first procedure, and this delay may be problematic especially in children. Moreover, despite repeated procedures, it may not be possible to completely and permanently cure the infection or eradicate all cholesteatoma. In these cases, the disease may be brought under control with repeated cleaning, after which cochlear implantation can go ahead. The patient should be informed of the increased risk and should be followed up vigilantly.

A single-stage procedure has been proposed that can be performed in the presence of cholesteatoma. The investigators argued that residual or recurrent cholesteatoma could occur at any time postoperatively and that there is no evidence of a second look for cholesteatoma before implantation prevents the implant from destructive recurrent cholesteatoma [22]. However, because of the potentially devastating risks of residual cholesteatoma in patients with a CI, and because diagnosis of cholesteatoma is complicated when obliterative techniques have been used, we would strongly advise against this policy.
Therefore, it is advisable to perform an intact canal wall tympanomastoidectomy or a radical mastoidectomy in patients with active CSOM, with or without cholesteatoma, followed by cochlear implantation in a second procedure once the ear is considered healthy.

\section{Radical cavities}

In the case of a radical cavity without active disease, after treatment of active disease in a radical cavity, or after creation of a radical cavity to resolve CSOM or cholesteatoma, special measures should be taken to protect the electrode in its passage through the cavity. The thin epithelial lining of the mastoid cavity is nearly always insufficient to protect any kind of implanted foreign body itself and, therefore, simply inserting the electrode under the epithelial lining leaves it vulnerable to exposure [7, 17, 18, 23-26, 29]. Although the exact rate of electrode extrusion and its possible consequences are not known, different techniques have been developed to protect the electrode.

It is possible to obliterate the cavity completely using abdominal fat $[8,10,14,19,20,25]$, a pedicled temporalis muscle flap [7, 9, 10, 17, 22], or a combination of both [27]. In the case of total obliteration, the Eustachian tube is obliterated as well and the external ear canal is closed as a blind sac. The cavity can be filled with bone dust, hydroxyapatite, and tricalcium phosphate. However, anatomical landmarks may be obscured and it may be necessary to drill at the time of cochlear implantation in a second procedure [20]. Moreover, hydroxyapatite and tricalcium phosphate carry the danger of infection and extrusion. The most suitable material to obliterate the cavity appears to be abdominal fat because of its resistance to necrosis and easy removal if cochlear implantation is performed later.

Entrapment of squamous epithelium is a potentially serious complication of obliterative surgery. Magnetic resonance imaging cannot be performed in these patients because of the magnet and the signal void around the implanted metal. High-resolution computed tomography can be performed, but findings are difficult to interpret and the predictive value is low. Because it is easier to distinguish the contours of cholesteatoma from air than from muscle or fat, it is considered preferable to leave the middle ear, the mastoid cavity, or both, aerated. Techniques have been developed to make this possible. One such technique, reconstruction of a new posterior canal wall [28], is not considered as advantageous because it involves complicated surgery, postoperative healing problems, and a risk of epithelial entrapment and will, therefore, not be discussed in detail.

Non-obliterative techniques involve drilling of the bed for the receiver-stimulator in a posterior position than in a standard procedure [18, 21, 26, 29, 30]. Furthermore, the 
groove for the electrode outside the cavity is shaped in a wave-like form [21, 29, 30]. As a result, the main length of the array lies outside the mastoid cavity, which reduces the risk of ulceration and necrosis of the covering flaps or skin. Subsequently, bone dust and fibrin glue can be used to cover the electrode in its tract within the cavity, resulting in a flat surface $[18,21,26,29]$. If necessary, this surface can be covered with cartilage [21, 29], temporal fascia [18, 21], a periosteal flap [29], a pedicled temporal muscle flap [18, 26], or any combination of the materials mentioned above. This results in a non-obliterated or partially obliterated cavity, which can then be covered with the epithelial lining of the cavity [21,30]. Although all these studies represented case reports of a maximum of three patients, together these studies have reported 14 patients that were implanted using this non-obliterative technique without any short- or longterm complications. Therefore, this technique seems to be a safe alternative for obliterative surgery in cochlear implantation, without the complicated radiologic diagnosis of cholesteatoma that is a consequence of obliteration.

Another non-obliterative technique involves the use of a vascularized temporalis fascia flap ('Hong Kong Flap') after (revision) mastoidectomy to cover the cavity and repair possible defects in the tympanic membrane after a first-stage procedure [31]. This technique was used in only one patient receiving a CI. Moreover, a staged procedure is advised to ensure viability and strength of the flap to protect the electrode by the time cochlear implantation is performed. Consequently, this technique is not suitable if a single procedure can be performed, such as when there is a healthy radical cavity.

More recently, a non-obliterative technique with closure of the external auditory meatus has been reported [11-13]. This technique can be performed in one or two stages, depending on whether active disease is present. Otoscopic examination of the cavity is not possible, but high-resolution computed tomography, for the detection of cholesteatoma, is. The mucosa, aeration of the mastoid and middle ear cavity, and Eustachian tube are left patent in this procedure. The latter is especially important because the Eustachian tube could be a pathway for infection that is difficult to diagnose without the possibility of otoscopic examination.

In patients with CSOM, with or without cholesteatoma or a radical cavity, it is also possible to place a CI via the middle fossa approach [32]. This technique avoids the need for multiple surgical procedures before implantation and contamination of the middle ear. However, this approach involves risks inherent to craniotomy and does not correct the underlying problems associated with CSOM or cholesteatoma. As such, it is not really an attractive alternative for transmastoidal procedures.

We would recommend performing a non-obliterative procedure after (revision) mastoidectomy to make the ear of a patient with a radical cavity suitable for implantation and to provide optimal electrode protection, preferably without meatal closure so that the cavity can be easily visualized.

\section{Otitis media in follow-up after cochlear implantation}

Even though the incidence of otitis media after implantation is lower than before implantion [3-5], it still occurs frequently in children. This decreased incidence might be due to rigorous preoperative management of otitis media, the decrease in the incidence of otitis media with age, and mastoidectomy performed during implantation [3-5]. If a patient with a CI, regardless of age, develops AOM, prompt treatment with antibiotics is recommended and most studies do not show any subsequent otitis-media related inner ear or intra-cranial complications [3-6]. Although antibiotics are usually used conservatively in the Netherlands, patients with a CI who develop an infection should be treated as early as possible because of the potential risks. Ventilating tubes are usually inserted before, during, or after implantation in children who are prone to otitis media, to prevent recurrent episodes of otitis media.

Can we conclude that infection after implantation is easy to treat and therefore not dangerous? Does this mean that the surgical measures taken to prevent recurrent infection in adults with CSOM are overdone? While AOM after implantation is effectively treated with antibiotics in patients of all ages, chronic disease, with or without cholesteatoma, is more hazardous and has obviously proved resistant to treatment in the past. For this reason, more extensive measures should be taken to prevent its recurrence after implantation. Therefore, all patients with CSOM, a radical cavity, tympanic perforation, or cholesteatoma should be followed up regularly. Patients with CIs should be seen by audiologists, but such patients with CSOM should also be seen regularly by an otolaryngologist, in order to diagnose, treat, or control a potential recurrent infection or cholesteatoma as early as possible.

A history of ear disease appears to significantly increase the risk of infection post-implantation [33]. In theory, this could also mean a higher risk of meningitis in these patients, since pathogens isolated from patients with meningitis after the perioperative period is the same as those that cause otitis media [34]. In the past, the incidence of bacterial meningitis was higher in patients with a CI than in the general population $[34,35]$. One type of implant seemed to contribute to this higher incidence of meningitis, probably due to its design and insertion, and has been removed from the market. However, candidates for CIs may also have predisposing factors that increase their risk of meningitis, such as a higher incidence of inner-ear malformations or cerebrospinal fluid leaks. Furthermore, patients whose deafness 
was caused by meningitis or labyrinthitis are also at increased risk of subsequent attacks of meningitis [36]. It has not yet been determined whether the implant, the preexisting risk factors, or a combination of both causes the increased rate of meningitis observed among individuals with CIs. More needs to be known about this, and to this end, the incidence of bacterial meningitis in patients with CIs should be compared to that of a comparable population of patients with deafness.

In the Netherlands, all patients with a $\mathrm{CI}$ are vaccinated against Haemophilus influenzae type B (Act-Hib, Aventis Pasteur MSD) and Streptococcus pneumoniae (Pneumovax, Aventis Pasteur MSD/Prevenar, Wyeth). We are aware that vaccination does not completely protect the patient against the development of meningitis and that a shift in pathogens may be expected, which makes it more likely that other pathogens will cause meningitis [37]. Nevertheless, vaccinating patients with a CI protects them from meningitis caused by a considerable number of pathogens and is therefore a valuable instrument against meningitis.

\section{Conclusion}

Cochlear implantation is possible in patients with otitis media if appropriate measures are taken to prepare the ear for implantation and to minimize potential risks, such as recurrence of the infection, entrapment of epithelium, extrusion of the device, and meningitis. An appropriate procedure should be based on the individual clinical presentation at the initial evaluation for implantation. This article provides a guideline to make this decision.

In summary:

- AOM Infection should be controlled before cochlear implantation. A single acute infection can be treated conservatively, whereas ventilation tubes may be more effective for recurrent $\mathrm{AOM}$.

- Tympanic membrane perforation Myringoplasty and cochlear implantation should be performed in a twostaged procedure.

- Purulent CSOM, cholesteatoma Intact canal wall tympanoplasty or radical mastoidectomy should be performed, followed by cochlear implantation in a second stage, using a non-obliterative technique.

- Radical cavity A non-obliterative procedure with optimal electrode protection should be performed, preferably without meatal closure.

In conclusion, cochlear implantation can be safely performed in patients with otitis media but it remains a challenging problem in which individualized, tailor-made precautions should be taken.
Conflict of interest statement The authors declare that they have no conflict of interest.

Open Access This article is distributed under the terms of the Creative Commons Attribution Noncommercial License which permits any noncommercial use, distribution, and reproduction in any medium, provided the original author(s) and source are credited.

\section{References}

1. Belal A Jr (1986) Contraindications to cochlear implantation. Am J Otol 7:172-175

2. Rovers MM, Schilder AGM, Zielhuis GA et al (2004) Otitis media. Lancet 363:465-473

3. Fayad JN, Tabaee A, Micheletto JN et al (2003) Cochlear implantation in children with otitis media. Laryngoscope 113:1224-1227

4. Luntz M, Teszler CB, Shpak T et al (2001) Cochlear implantation in healthy and otitis-prone children: a prospective study. Laryngoscope 111:1614-1618

5. Luntz M, Teszler CB, Shpak T (2004) Cochlear implantation in children with otitis media: second stage of a long-term prospective study. Int J Pediatr Otorhinolaryngol 68:273-280

6. Kennedy RJ, Shelton C (2005) Ventilation tubes and cochlear implants: what do we do? Otol Neurotol 26:438-441

7. Axon PR, Mawman DJ, Upile T et al (1997) Cochlear implantation in the presence of chronic suppurative otitis media. J Laryngol Otol 111:228-232

8. Incesulu A, Kocaturk S, Vural M (2004) Cochlear implantation in chronic otitis media. J Laryngol Otol 118:3-7

9. Babighian G (1993) Problems in cochlear implant surgery. Adv Otorhinolaryngol 48:65-69

10. Takahashi H, Naito Y, Fujiki N et al (2000) Cochlear implant surgery in ears with chronic otitis media. Adv Otorhinolaryngol 57:93-95

11. El-Kashlan HK, Arts HA, Telian SA (2002) Cochlear implantation in chronic suppurative otitis media. Otol Neurotol 23:53-55

12. El-Kashlan HK, Arts HA, Telian SA (2003) External auditory canal closure in cochlear implant surgery. Otol Neurotol 24:404-408

13. El-Kashlan HK, Telian SA (2004) Cochlear implantation in the chronically diseased ear. Curr Opin Otolaryngol Head Neck Surg 12:384-386

14. Bendet E, Cerenko D, Linder TE et al (1998) Cochlear implantation after subtotal petrosectomies. Eur Arch Otorhinolaryngol 255:169-174

15. Donnelly MJ, Pyman BC, Clark GM (1995) Chronic middle ear disease and cochlear implantation. Ann Otol Rhinol Laryngol Suppl 166:406-408

16. Roehm PC, Gantz BJ (2006) Cochlear implant explantation as a sequel of severe chronic otitis media: case report and review of the literature. Otol Neurotol 27:332-336

17. Kim CS, Chang SO, Lee HJ et al (2004) Cochlear implantation in patients with a history of chronic otitis media. Acta Otolaryngol 124:1033-1038

18. Himi T, Harabuchi Y, Shintani T et al (1997) Surgical strategy of cochlear implantation in patients with chronic middle ear disease. Audiol Neurootol 2:410-417

19. Gray RF, Irving RM (1995) Cochlear implants in chronic suppurative otitis media. Am J Otol 16:682-686

20. Gray RF, Ray J, McFerran DJ (1999) Further experience with fat graft obliteration of mastoid cavities for cochlear implants. J Laryngol Otol 113:881-884

21. Marangos N, Laszig R (1997) Cochlear implant surgery and radical cavities. Adv Otorhinolaryngol 52:147-150 
22. Basavaraj S, Shanks M, Sivaji N et al (2005) Cochlear implantation and management of chronic suppurative otitis media: single stage procedure? Eur Arch Otorhinolaryngol 262:852-855

23. Webb RL, Lehnhardt E, Clark GM et al (1991) Surgical complications with the cochlear multiple-channel intracochlear implant: experience at Hannover and Melbourne. Ann Otol Rhinol Laryngol 100:131-136

24. Cohen NL, Hoffman RA, Stroschein M (1988) Medical or surgical complications related to the Nucleus multichannel cochlear implant. Ann Otol Rhinol Laryngol Suppl 135:8-13

25. Pasanisi E, Vincenti V, Bacciu A et al (2002) Multichannel cochlear implantation in radical mastoidectomy cavities. Otolaryngol Head Neck Surg 127:432-436

26. Kiefer J, von Ilberg C (1997) Special surgical problems in cochlear implant patients. Adv Otorhinolaryngol 52:135-139

27. Hamzavi J, Baumgartner W, Franz P et al (2001) Radical cavities and cochlear implantation. Acta Otolaryngol 121:607-609

28. Tamura Y, Shinkawa A, Ishida K et al (1997) Cochlear implant after reconstruction of the external bony canal wall and tympanic cavity in radically mastoidectomized patients with cholesteatoma. Auris Nasus Larynx 24:361-366

29. Manrique M, Cervera-Paz FJ, Espinosa JM et al (1996) Cochlear implantation in radical cavities of mastoidectomy. Laryngoscope 106:1562-1565

30. Karatzanis AD, Chimona TS, Prokopakis EP et al (2003) Cochlear implantation after radical mastoidectomy: management of a challenging case. ORL J Otorhinolaryngol Relat Spec $65: 375-378$

31. Tong MC, van Hasselt CA (1997) Cochlear implant surgery in a modified radical mastoidectomy cavity reconstructed utilizing the Hong Kong vascularized temporalis fascia flap technique. Adv Otorhinolaryngol 52:151-154

32. Colletti V, Fiorino FG, Carner M et al (2000) New approach for cochlear implantation: cochleostomy through the middle fossa. Otolaryngol Head Neck Surg 123:467-474

33. Cunningham CD 3rd, Slattery WH 3rd, Luxford WM (2004) Postoperative infection in cochlear implant patients. Otolaryngol Head Neck Surg 131:109-114

34. Reefhuis J, Honein MA, Whitney CG et al (2003) Risk of bacterial meningitis in children with cochlear implants. N Engl J Med 349:435-445

35. US Food Drug Administration (2003) Cochlear implants and meningitis. FDA Consum 37:35

36. Bluestone CD (2003) Prevention of meningitis: cochlear implants and inner ear abnormalities. Arch Otolaryngol Head Neck Surg 129:279-281

37. Sanders L (2005) Otitis media and cochlear implantation-pointers and pitfalls. Immunology and infection. Presented at the 5th extraordinary international symposium on recent advances in otitis media, Amsterdam, the Netherlands, 24-27 April 2005 